\title{
INDUSTRIAL APPLICATION OF INTERNET OF THINGS
}

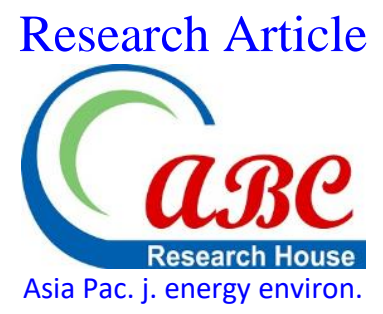

Naresh Babu Bynagari

Andriod Developer, Keypixel Software Solutions, 777 Washington rd Parlin NJ 08859, Middlesex, USA

*Email for Correspondence: bynagari.gs@ gmail.com

Abstract

'Industrial application of Internet of Things' deals with the application of Internet of things to produce industrial services. It analyzed how industries can carry out multiple services with function remotely using IoT-connected devices. The several benefits and drawbacks to the application of IoT services were also investigated. The IoT is a network of connected systems and smart devices that use encoded networks like sensors, processors, and interactive hardware to receive, send and store data. The utilization of IoT for industrial functions will significantly improve industrial output, and in the future, more industries will come to apply IoT devices and systems for greater efficiency.

Key words

Internet of things (IoT), Computer Network, Servers Network, Sensors Analytics

\section{INTRODUCTION}

Internet of Things (IoT) refers to a network of interconnected computer systems. They can be registered animals or humans with UIDs (unique identifiers). The connected systems can be mechanical or digital and automated devices that are embedded with UIDs. Transfer of data in the IoT is possible within the connected devices without the needing man to man or man to computer communication. To be a Thing in the IoT, there must be a unique identifier. This could be a biochip with a transponder implanted in an animal for tracking or a health implant in a human with a chip (Taher-Uz-Zaman et al., 2014). A machine with advanced artificial intelligence can detect faults for maintenance, like a sensor in cars that can detect faults and warn the driver of such faults.

Industrial IoT deploys edge technology and smart sensors interconnected within the industrial environment and settings like manufacturing and production, energy, administration, management, etc. This system will allow businesses to run their affairs smoothly and use gathered data to enhance operations.

This area is rapidly evolving as companies such as Google and Microsoft set aside significant resources to improve their IoT platforms.

\section{THE INTERNET OF THINGS}

The IoT network comprises systems and a network of web-connected intelligent devices that employ encoded networks like sensors, processors, and interactive hardware to receive, send and store data. The network in the IoT uses the stored data. To use the stored data, the IoT device sends out the collected sensor information through a connection with a gateway on the IoT or through other means such as using edge tools where data is either sent for analysis on the local network or in the cloud. Connected devices on the IoT network interact and use the shared information they acquire to solve problems. The interactions within the network are done without human supervision or interference, and most of the job is carried out by the devices (Paruchuri, 2015). Even though humans may communicate with the devices to set them up, writing scripts for protocols and commands provide access to information.

The Different applications used by IoT decide the protocol and system of connection, communication, and network which the things or devices are to use. Artificial Intelligence (AI) and machine learning in IoT improve data collection and processing by the connected devices in a network system. Machine learning makes it dynamic. 


\section{The lot Presents Several Advantages}

Some of the benefits are for industries, while others apply to several types of industries. We would investigate the following IoT advantages to business enterprises.

- IoT monitors the process of the business.

- It makes the customer experience (CX) more efficient.

- IoT through automated processes helps save time and reduce cost.

- IoT through the connected network of data helps improve employees' output.

- It also connects various business models

- Through data sharing allows businesses to make better decisions.

- In saving time and cost helps improve revenue.

The connected network system of the IoT allows companies to select a proper technique for their business as it gives them the information and tools for better choice. IoT uses sensors and other devices to manufacture, transport data. Through machine learning, IoT manages infrastructure within the organization making these industries more digitalized and transformed (Neogy \& Paruchuri, 2014).

For instance, IoT can help improve farming and make it easier to farm in the agricultural industry. Information and data about the weather can be collected by sensors connected to the Internet of Things which detect rainfall, temperature, and the content of the soil, along with other pieces of information that may help improve the industry. IoT through automated machines would help improve farming methods. Also, the need to manage machines, pieces of equipment, and infrastructure can be solved using IoT.

For instance, structures and buildings could be monitored by sensors that can detect changes in events and malfunctioning of infrastructures and equipment within the industries. These management techniques are costeffective, save time, and increases the quality of workflow.

Also, IoT can be used to automate electrical systems by manipulating and monitoring systems in a home. Also, people can be monitored by IoT in an intelligent City to limit unnecessary cost and energy use. The business, Heath, financial, and manufacturing industries can all be influenced by IoT.

\section{DOWNSIDES OF IOT}

IoT applications cut across many technologies and advanced internet worlds, ranging from consumer to industrial sector. For example, smart homes with smoke detectors, Artificial Intelligence security systems, and connected devices can be controlled through computers or smartphones.

- The risk of data and information theft by hackers. The growth of IoT means an increase in the connection of devices and the growth of data sharing between connected devices. The impact and hood of a hack would also be huge.

- The volume of data used would also increase. This would make it a more complicated task to manage the large inflow of data in IoT by persons and content management systems.

- Through the shared network, whenever a bug affects a system, there is the tendency that it may also affect all the devices connected and corrupt them.

- IoT lacks an international standard for the compatibility of the device. Issues may come up due to the difference of the manufacturing devices to communicate with each other.

Billions of devices are brought into the system through the IoT, establishing a connection on the internet and IoT network. It brings about the need to use even more data and a bigger database. The data and data must be kept secured from hacks or attacks (Donepudi, 2014). The growing database of devices on the IoT has been a cause of fear and concern the security and privacy of data remains a big issue. 


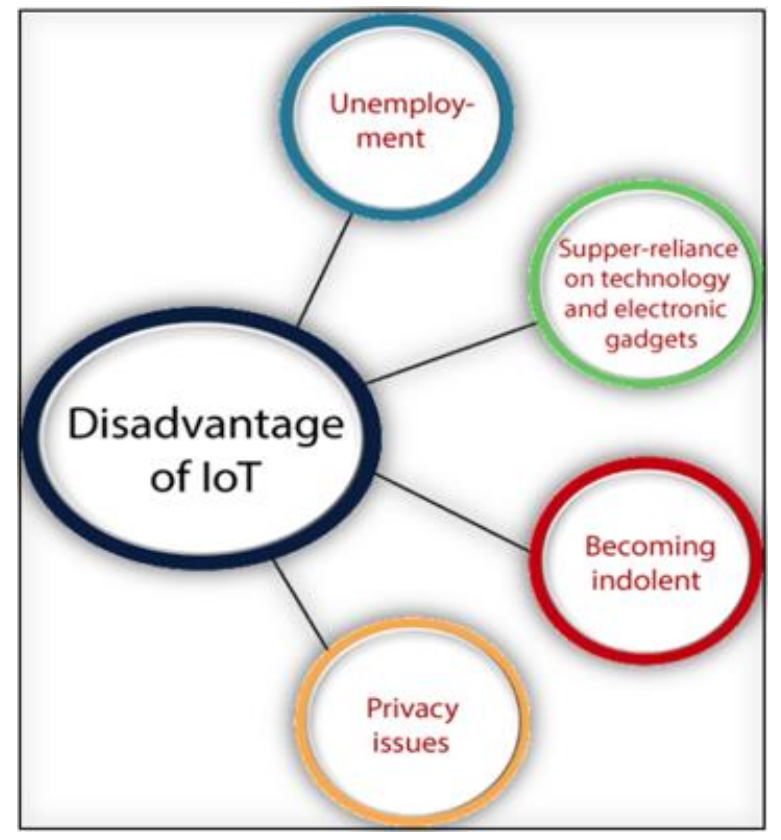

Figure 1: Disadvantages of IoT (Source: tutorialandexample.com)

\section{Privacy Issues Associated With loT}

IoT applications cut across many technologies and advanced internet worlds, ranging from consumer to industrial sector. For example, smart homes with smoke detectors, Artificial Intelligence security systems, and connected devices can be controlled through computers or smartphones.

Billions of devices are brought into the system through the IoT, establishing a connection on the internet and IoT network. It brings about the need to use even more data and a bigger database. The data and data must be kept secured from hacks or attacks (Donepudi, 2014). The growing database of devices on the IoT has been a cause of fear and concern the security and privacy of data remains a big issue.

A popular IoT threat was in 2016. The Mirai attack used the botnet to bypass Dyn. Dyn provides hosting and servers for domain names and websites. The hack affected a lot of websites for a long time. Many view it as the longest distributed denial-of-service (DDoS) hacks ever witnessed. The hackers used poorly secured devices on the IoT network to gain access to the network. As mentioned before, the interconnected nature of the IoT network makes it easier to attack the system. A weak device on the network could expose the whole network and database to threats. A hacker needs to use a vulnerable device, manipulate the data, and gain access to make it useless.

Regular updates help prevent security breaches. Unfortunately, most manufacturers don't update their products on time. This makes it easier to hack the device.

Devices on the network from time to time usually request for personal data of users. This could include their biodata, addresses, bank account details, and so on. This data becomes a target for hackers.

Privacy on the IoT is another factor and cause of significant concern of users. Companies that produce IoT devices can use them to gather personal data and sell them too.

\section{INDUSTRIAL APPLICATION OF INTERNET OF THINGS}

The industrial application of the Internet of Things uses the Internet of things connected intelligent devices for industrial applications for functions such as predictive maintenance, monitoring equipment remotely, automation, etc. The application of IoT in industries is a more advanced internet of things. From the agricultural sensor systems to vehicle construction, supply chain robotic, and other areas of consumers and commercial applications, IoT smart connected devices have been deployed to enhance performance and output. The Industrial Internet of things applications and devices are usually deployed in harsh environments (Bynagari, 2015). There are other small industrial applications of IoT devices such as meters and heavy-duty machines used in industries that are affected by harsh environmental conditions like excessive heat and cold. 


\section{Industrial Internet of Things}

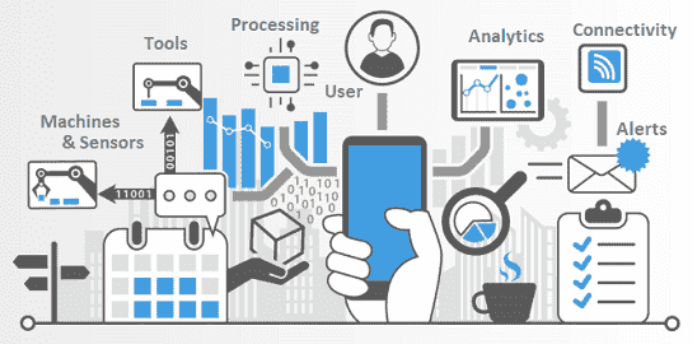

Figure 2: IIoT (Source: rocknetworks.com)

The application of IoT devices in industrial activities is for data gathering and transmission, monitoring changes, flows, or volume, automating processes for higher accuracy, and reducing workplace hazards. However, the Industrial Internet of Things is not just about automating procedures, and there have been some changes and projects which has allowed even more activities for industrial IoT (Ganapathy, 2015). As a result, the cross-industry has the highest growing categories of IoT applications.

Industries have as used the application of the Internet of Things in industries to transform industrial operations. In this sense, several Industrial IoT has been used to develop smart factories and applied in other industrial functions.

Several technological frameworks enable industrial Internet of Things applications on different layers. Frameworks such as edge and fog computing are the most popular in applying the Internet of Things in industrial activities. In addition, there are multiple networks and tools for connectivity.

\section{Ways of Industrial Internet of Things application}

Digital Transformation. The industrial application of the Internet of things allows for a network of devices, machinery, and sensors on the Internet to gather and analyze data. It uses the collected data and information for continuous system enhancement. There is numerous industrial Internet of things frameworks and systems that are currently available. They have enabled many industries to use the technology to enhance productivity and improve cost and profits.

Major players have been adopting the Industrial IoT marketing system, which is a constantly increasing market. Most research shows that the Industrial application of IoT has transformed the industrial sector to allow greater digitization for best results. Digital transformation of industries is an important strategic objective for industries. Businesses and organizations need to be aware of the great advantage of IoT applications in the industrial sector. The IoT application in the industrial sector develops processes that will be useful in the future.

\section{a) Remote Monitoring and Management.}

Automated equipment management and monitoring are some of the main applications of IoT in industries. It allows a central system to control and monitor the entire company's operations.

Through this feature, several structures and machines can be remotely manipulated from different locations across the globe. This gives the companies the advantage of controlling and monitoring the advances in their productivity in real-time and at the same time can study and carry out analysis on historical data that is gathered which are connected to the processes (Bynagari, 2014). This data gathering aims to use the data to enhance processes and develop an environment in which decisions are made based on quality information. Some automated and remote equipment monitoring and management in different industries include: 


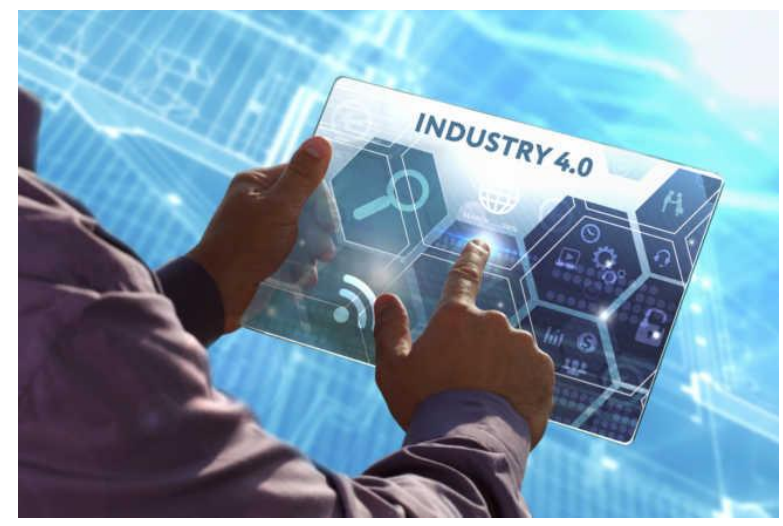

Figure 3: Remote monitoring (Source: iiot-world.com/industrial-iot)

- Tank monitoring (oil and Gas sector): In the past, to check the oil or fluid levels in tanks, some of the most common practices involve using a dipstick or throwing stones at the tank. These methods can be enhanced immensely with the application of IoT technologies in Industries. Tanks must be monitored to eliminate overflow at production and manufacturing, attracting huge fines from clean-up expenses. Although monitoring onsite tanks with chemicals infused into pipelines to reduce corrosion is essential for routine maintenance. Industries can introduce an industrial IoT to lessen risk and enhance the efficiency of operations. For example, an automated tank monitoring system can send tank level data reading on a schedule (Hourly, daily, weekly, and so on) to enable monitoring by field managers and allow them to manage deliveries and pick-ups. It also allows them to carry out preventive maintenance using the received data and level information. This system also prevents the cost of sending staff or persons to check tank levels, whether it is low as personnel are sent when needed.

- Flow monitoring in the agricultural industry: a popular way of distributing water in agriculture is center pivots. Water passes through a pipe and is distributed through a sprinkler that rotates at the center in circles. Leakages in the center pivots and irrigation channels or pipes can incur considerable repairs and resource loss. The use of remote monitoring and maintenance for pressure and movement can enhance detecting issues in the pipeline and flow chain. IoT devices can detect and set off alarms early in the event of unplugged heads, leaks, and even improper fittings. This can help mitigate issues, maintain crop growth, and reduce the cost of repairs.

- Monitoring chemical processes: in refineries, for example, where chemical levels must be constantly monitored, the use of IoT devices is quite important. This monitoring must not be from a huge distance. It can be just a hundred feet or a football pitch away. Monitoring has been done by installing a wireline in the facilities. A significant challenge faced in using wirelines is that the cables are expensive and may be affected by corrosive agents in the environment. Other environmental hazards like weather storms or earthquakes may also affect it. Thousands of IoT sensors may be installed in processing plants to collect and transmit data. For instance, sensors may be used to monitor and control a chemical batch to avoid explosion or spillage. Most companies are currently looking at IoT for industrial to enhance automation and monitoring of industrial processes. The IoT carries out analysis and records them in a cloud-based system for analysis and provides alerts that can be remotely accessed.

\section{b) Predictive Maintenance}

This type of maintenance detects machine maintenance needs and gives alerts to avoid considerable damages to a system. It is a preventive approach to maintenance that allows for early detection of faults or failures, resulting in substantial financial loss from repair costs and work time. This is part of why data gathering, analyzing, and management systems are implemented.

Preventive maintenance systems are part of the most useful Industrial Internet of Things applications. It functions using sensors that can send signals in the event of risk factors after being installed on the operating platforms and the machines. For instance, sensors used in monitoring robots and machines transmit data to the systems and analyses the data in real-time and deploys enhanced algorithms that can give alerts of issues like overheating, vibrations, and other factors that exceed the parameters considered as usual. Some examples of IIOT predictive maintenance application in numerous industries including: 


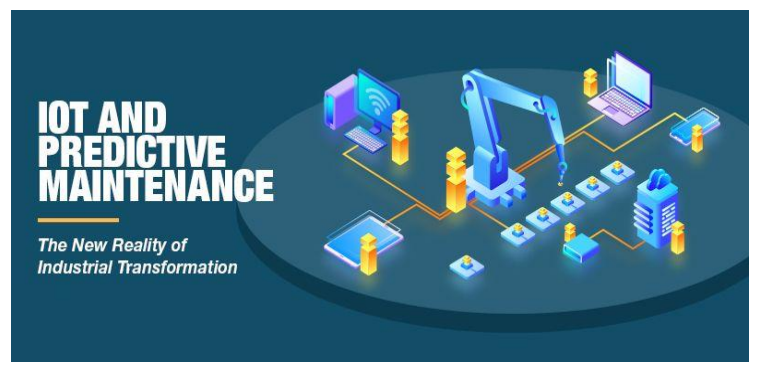

Figure 4: Preventive maintenance (Source: hiotron.com)

- Process monitoring: in mining, for instance, a giant conveyor belt carries earth from one extraction point. The transportation of these things could take very long. Sometimes over a mile or two. In the event of fault or problems to the conveyor belt during use, it can result in substantial financial losses of up to millions. Sensors that work $24 / 7$ can be installed on the conveyor for data gathering points. The data may then be transmitted through a radio device to a gateway before sending it to a remote monitoring application. An example of such a system is Digi Remote Manager, which can help mines and queries avoid unwanted downtime.

- Maintenance of Equipment - companies in elevator production, may want to ensure that their users get the best quality service. So, companies install hundreds of thousands of sensors that carry out preventive maintenance on these elevators instead of waiting for faults or failures to come up. The sensor transmits data points using a gateway to a cloud environment that enables automated alerts to personnel and provides insights.

- Asset monitoring: Energy management companies help provide solutions to companies by developing some IoT tools to help the industry identify problems before they result in more significant issues. For example, the use of IoT-connected devices in monitoring equipment, identifying, and reporting points of failure of things like a hydraulic hose to prevent equipment from breaking down and the company incurring an unbelievable loss.

- Improved implementation: IIoT application brings about essential information sharing, which can help improve the processes in an industrial setup. Business systems from the quality to the processes and manufacturing can be remotely carried out quickly and automatically. This feature also changes the tempo at which the changes and improvements can be applied in operational and business intelligence.

\section{c) Inventory Management}

The industrial application of IoT systems allows companies to monitor inventories automatically and satisfy whether the proper plans are being followed and gives alerts in case of variation. This is also an essential application of the Internet of things in industrial functions to maintain a consistent and good workflow.

\section{d) Integrity control.}

Another important IoT application in the industry is the quality control feature that allows personnel to manage and monitor manufactured products $24 / 7$. From the raw material stage, when they are used being transported using a smart tracking system to the monitoring of the reaction and sentiments of the end-user after the product is used.

All this data is essential when analyzing company efficiency and applying the important changes when faults are noticed. This aims to enhance service and processes by detecting problems in the production chain on time. According to research, this will also prevent risks in highly delicate industries like pharmaceuticals and so on.

\section{e) Supply chain enhancement}

Supply chain optimization allows for real-time on-the-go data on the status of a company's supply chain. This feature makes it possible to pinpoint the issues limiting the processes, making them ineffective, resulting in the loss.

\section{f) Plant Safety enhancement}

Internet of Things connected devices can generate real-time information on the status of the plant or factory. This may be achieved through equipment damage monitoring and how common specific illnesses are in the company, among other factors (Donepudi, 2015). Through this feature, a company can monitor and keep track of the conditions and prevent hazardous events that may threaten workers' health and life. This will boost workers' health and improve safety in the working environment and result in better productivity and employee motivation. 


\section{g) Automated modification}

Most industries and companies are turning to artificial intelligence and automating machines to improve the behavior of equipment based on conditions. For instance, during hot summer, the wind usually brings down to hot temperatures. However, too much wind can lead to the destruction of equipment in any industry. Windmills, agricultural and solar installations can be destroyed by too much wind. During storms with heavy winds in solar fields, wind speed and other adverse weather conditions can be monitored in weather stations to detect when wind speeds will get to a damaging level (Vadlamudi, 2015). Wireless transmissions from a gateway can transmit commands that allow the solar panels to take a better position that can help mitigate damages.

\section{BARRIERS FACED IN THE APPLICATION OF THE INTERNET OF THINGS IN INDUSTRIES}

The application and adoption of IoT, despite the barriers, is positioned to grow and develop as more companies adopt it. Some of the challenges faced in the adoption of IoT in industries including:

- Data integration: data collected from the industrial output are usually complex, according to research by infographic (2016). Data complexity is part of the significant challenges of this era. The numerous forms, the massive volume of data (mostly from big industries), difference in date frequency, and complicated data relations. Integration of data with systems is one of the significant barriers to the application of IoT frameworks in industries. There is the constant challenge of moving data from the raw form to the form in which it can be used business-wise. Data, insights, and knowledge within the sharing space are where revenue-generating opportunities will be.

- Inadequacy of the right skills and experienced personnel: According to research, lack of skills is also one of the primary reasons companies in the industrial sector are not ready for IoT application in industries. Thirty-six percent of respondents suggested lack of access to quality skills and experienced personnel as a problem to the application of IoT in industrial applications. The lack of qualified and highly skilled workers that can handle IoT systems in industries can be solved by looking outside to get the right skills. The importance of Industrial Internet of things application in this age of information and technology cannot be overemphasized. The lack of skilled personnel problem may be avoided by outsourcing the operation of IoT to skilled experts and professionals. Furthermore, networks, systems, and frameworks must be put in place as no organization can do it on its own.

- Cyber and data security threats: This is also a significant challenge for the application of IoT in industrial operations. Most organizations tend to keep some data and information secret and show great concern for data safety. The numerous forms of data and cybersecurity threats have become a considerable challenge to the adoption of industrial IoT. Currently, companies providing industrial IoT services usually provide hybrid IoT connectivity solutions for use in the industrial sector. Some of these services include Cellular IoT, LPWAN (Low Power Wide Area Networks), and so on for industrial connectivity solutions, and so on.

\section{Conclusion}

The Internet of things connects all things (devices like sensors) on its network using a wireless network on webservers. The development and advancement of the world bring about more need for technology to solve problems. Industrial application of the Internet of things through connected devices and sensors would help reduce some industrial risk factors and enhance output. Industrial IoT will make industrial works easier.

There is bound to be more application of IoT devices in industries in the future as more companies and industries apply IoT devices to automate functions. As a result, more enterprises will become digitalized through this application.

\section{References}

Bynagari, N. B. (2014). Integrated Reasoning Engine for Code Clone Detection. ABC Journal of Advanced Research, 3(2), 143-152. https://doi.org/10.18034/abcjar.v3i2.575

Bynagari, N. B. (2015). Machine Learning and Artificial Intelligence in Online Fake Transaction Alerting. Engineering International, 3(2), 115-126. https://doi.org/10.18034/ei.v3i2.566

Donepudi, P. K. (2014). Technology Growth in Shipping Industry: An Overview. American Journal of Trade and Policy, 1(3), 137-142. https://doi.org/10.18034/ajtp.v1i3.503

Donepudi, P. K. (2014). Voice Search Technology: An Overview. Engineering International, 2(2), 91102. https://doi.org/10.18034/ei.v2i2.502 
Donepudi, P. K. (2015). Crossing Point of Artificial Intelligence in Cybersecurity. American Journal of Trade and Policy, 2(3), 121-128. https://doi.org/10.18034/ajtp.v2i3.493

Ganapathy, A. (2015). AI Fitness Checks, Maintenance and Monitoring on Systems Managing Content \& Data: A Study on CMS World. Malaysian Journal of Medical and Biological Research,2(2), 113-118. https://doi.org/10.18034/mjmbr.v2i2.553

Neogy, T. K., \& Paruchuri, H. (2014). Machine Learning as a New Search Engine Interface: An Overview. Engineering International, 2(2), 103-112. https://doi.org/10.18034/ei.v2i2.539

Paruchuri, H. (2015). Application of Artificial Neural Network to ANPR: An Overview. ABC Journal of Advanced Research, 4(2), 143-152. https://doi.org/10.18034/abcjar.v4i2.549

Taher-Uz-Zaman, M., Ahmed, M. S., Hossain, S., Hossain, S., \& Jamal, G. R. A. (2014). Multipurpose Tactical Robot. Engineering International, 2(1), 21-27. https://doi.org/10.18034/ei.v2i1.204

Vadlamudi, S. (2015). Enabling Trustworthiness in Artificial Intelligence - A Detailed Discussion. Engineering International, 3(2), 105-114. https://doi.org/10.18034/ei.v3i2.519 